\title{
Increased Nuchal Translucency: Is Advanced Sequencing the Answer? A Mini Commentary
}

\author{
Asha Talati ${ }^{1}$ and Neeta Vora ${ }^{2}$ \\ ${ }^{1}$ University of North Carolina System \\ ${ }^{2}$ Affiliation not available
}

September 25, 2021

Title: Increased Nuchal Translucency: Is Advanced Sequencing the Answer? A Mini Commentary.

Asha N. Talati ${ }^{1}$, Neeta L. Vora ${ }^{1}$

Affiliations:

${ }^{1}$ Department of Obstetrics and Gynecology, Division of Maternal-Fetal Medicine University of North Carolina School of Medicine, Chapel Hill, NC

Corresponding Author:

Asha N. Talati MD MS

3010 Old Clinic Building, Campus Box 7516

Chapel Hill, NC, 27599

Telephone: 9199662229

Email: asha_talati@med.unc.edu

Short Running Title: Increased NT and Sequencing

\section{Acknowledgements:}

We would like to acknowledge the University of North Carolina Division of Maternal Fetal Medicine and Division of Reproductive Genetics for their support in our ongoing work in incorporation of exome and whole genome sequencing into prenatal diagnosis.

Disclosure of Interests : The authors have no relevant disclosures.

Contribution to Authorship: AT and NT were both equally involved in conception, planning, and writing of this mini commentary.

Ethics Approval: n/a

Funding : NICHD K23HD088742 (PI: Vora)

Increased nuchal translucency (NT) on first trimester ultrasound poses a diagnostic and prognostic dilemma. Given association with chromosomal changes and other structural anomalies, an isolated increased NT is an indication to pursue genetic diagnosis. Early diagnosis may aid decisions for pregnancy management as a chromosomal abnormality is identified in $30 \%$ of cases. However, with a normal chromosomal analysis, the 
absence of other anomalies, and resolution of increased NT, a healthy infant with no major anomalies results in over 95\% of cases (Bilardo et. al Ultrasound Obstet Gynecol, 2007; p11-8).

For the $70 \%$ of patients who will receive a negative chromosomal analysis, the question arises: how can we best use advanced sequencing to provide answers for parents, while reducing the risks of uncertainty and decisional conflict? Prior studies have demonstrated that, at this time, diagnostic yield is highest in the setting of negative chromosomal microarray and presence of multiple anomalies (Lord et. al Lancet2019; p.747-57). However, beyond nomenclature and interpretation, there have been few advances on how to best incorporate sequencing technology into the prenatal fold. Prior studies have described institutional approaches to integrating exome sequencing into the diagnostic paradigm (Vora et al, Genetics in Medicine, 2020; p 1-8), and few studies have highlighted the importance of extensive counseling and need for additional post sequencing support given the possibility of test-related anxiety and distress (Talati et. al, Genetics in Medicine , 2020). As such, there is still limited guidance on how to seamlessly incorporate sequencing into the diagnostic algorithm, particularly in specific settings like increased NT.

In their paper, Mellis et. al attempt to answer this question. Their group evaluated the utility of exome sequencing for (1) isolated increased NT, or (2) increased NT in the context of multiple fetal anomalies diagnosed in the first trimester or later in pregnancy. Prenatal exome sequencing had a low diagnostic yield $(<2 \%)$ for isolated increased NT but the authors found that the presence of additional anomalies in the first or second trimester dramatically increased diagnostic yield, consistent with findings from other cohorts. Additionally, increasing size of the nuchal translucency correlates with improved likelihood of diagnosis, with diagnostic yield similar to the presence of other anomalies when NT was $>7.5 \mathrm{~mm}$. The authors conclude that prenatal exome sequencing may not be appropriate for truly isolated increased NT, but may be considered when other anomalies are present or emerge to maximize likelihood of diagnosis (Mellis et. al, BJOG , 2021). The study by Melliset. al contributes to a growing body of literature that informs the clinical scenarios that benefit from sequencing strategies. However, there are significant challenges specific to variant interpretation in the setting of limited prenatal phenotypes and the possibility of uncertain results. Although this study provides preliminary information, it also reminds us of the significant work ahead to understand how the information we present can impact pregnancy experience and management, and most importantly, how we must first do no harm. 